\title{
Changes in family functioning for cancer patients undergoing surgery for the first time and their spouses
}

\author{
Miki Nakamori, Toyoe Taguchi \\ School of Nursing Science, Meiji University of Integrative Medicine, Kyoto, Japan
}

Correspondence: Miki Nakamori. Address: Department of Adult and Gerontological Nursing, School of Nursing Science, Meiji University of Integrative Medicine, Hiyosi-cho, Nantan-shi, Kyoto, 629-0392, Japan. Email:

m_nakamori@meiji-u.ac.jp.

Received: February 18, 2012

Accepted: March 21, $2013 \quad$ Online Published:

DOI : $10.5430 /$ jnep.v3n7p159

URL: http://dx.doi.org/10.5430/jnep.v3n7p159

\begin{abstract}
The purpose of the present study, involving patients undergoing surgery under general anesthesia for the first time and their families, was to examine changes in family functioning during the period before surgery and one month after it. Cancer patients who had never received surgical treatment and their families underwent the FFFS (Feetham Family Functioning Survey) Japanese version I. For both patients and their spouses, the level of importance of family functioning before the patients underwent surgery was higher, compared to after they received treatment. One month after surgery, the importance level of family functioning associated with "the relationship between the family and subsystems" for patients was significantly lower, and the importance level associated with "the relationships between the family and each family member" for spouses was significantly lower. This was because patients undergoing cancer surgery for the first time placed more importance on the relationship with medical professionals (as a subsystem), rather than family members. To cope with the crisis situation - patients undergoing their first surgery for cancer, their spouses desired to help each other with patients and other family members. When a cancer patient receives surgical treatment for the first time, it is important for health care professionals to establish appropriate relationships with them. Health professionals, nurses in particular, are also required to take time to provide intervention, including a proper explanation of treatment and support. It is necessary for medical professionals to help patients' spouses accept the crisis situation - their families undergoing cancer surgery for the first time, confirm their roles, and support the patients.
\end{abstract}

\section{Key words}

Cancer patient and spouse, Family functioning, Perioperative period nursing, FFFS Japanese version I

\section{I ntroduction}

In Japan, efforts to shorten the mean hospitalization period are in progress ${ }^{[1]}$ with the aim of reducing the medical care expenditure, and problems, such as difficulty providing appropriate guidance, the burden on family members, and their anxieties, as well as the importance of interacting with patients and their families, have been pointed out ${ }^{[2]}$.

Among various definitions of the family, Morioka ${ }^{[3]}$ defined the family as "a group for the pursuit of happiness consisting of a small number of close relatives, such as married couples, parents and children, brothers, and sisters, who have formed 
an emotional bond with each other”. A family is a group for communal living, and has multifaceted functions, including reproduction, economy, protection, education, health, and amusement, rather than a single specific one, to pursue happiness. However, although the basis of families is still married couples, their forms have become more complex with time and the number of one-household families has become smaller as a result of the aging population combined with the declining birth rate and the trend toward nuclear families. Whereas the majority of these family functions have been incorporated into social and other systems or become weakened, it has been pointed out that their mental health function, which helps family members consult with each other regarding worries and personal problems, has improved ${ }^{[3]}$. However, as smaller numbers of families mean a significant decrease in human resources that can be used to handle a crisis or address risks, there has been a decrease in the comprehensive function of families in recent years. A decrease in family functioning inhibits the development of subsystems among the family members, which, in turn, will deteriorate the functions that influence their emotions ${ }^{[4]}$. Considering these characteristics of modern families, it is not difficult to predict that the hospitalization of a family member and other serious events in daily life will affect the familial relationship and functions. In particular, as surgery is a life-threatening, invasive treatment method, it is expected to cause significant changes in family relationships and functioning. It is necessary to accurately assess these changes, and provide nursing intervention according to changes in the relationships between patients and their families and family functioning. It is important to develop effective and efficient nursing intervention methods for patients undergoing surgery and their families, and support family members to enhance their capabilities, rather than providing support unilaterally. To this end, a clear point of view is required to assess the relationship between patients and their families. Most previous studies of family functioning focused on the parents of pediatric patients ${ }^{[5]}$ and family members providing nursing care for the elderly ${ }^{[6]}$. Although some studies have involved the families of adult patients who were informed of cancer or undergoing treatment ${ }^{[7]}$ and kidney transplantation ${ }^{[8]}$, most of them covered only family members, and examined and evaluated family functioning at one point instead of over time. Therefore, it is also necessary to conduct studies of late-middle-aged and young elderly patients, who play important roles in the family and society, family members, and the functions of these families. The implications to nursing of this study is considering it as the key which considers concrete nursing care of family nursing of the operation term.

In 1976, researchers including Doctor Feetham developed the FFFS (Feetham Family Functioning Survey) designed to determine the function of families who have children with congenital myelatelia. The FFFS has been used in research of families with healthy children, families with children who have health problems, and families with adult children and adults with health problems, families with mothers with high-risk pregnancies, and refugee and immigrant families. Since the 1988 publication there been 28 published reports of use of the FFFS search for CINAL plus. These studies support the validity and reliability of the FFFS as a measure of the family functioning construct. The FFFSI is the Japanese version of the FFFS questionnaire developed in 2000 by Hohashi ${ }^{[9]}$. In the FFFSI, subjects are usually adult couples, such as husbands and wives or fathers and mothers, although husbands or wives alone can be subjects. The FFFS, which focuses on the relationship between a husband and wife, rather than the complex function of a family, was developed to examine the interaction between a family and its surrounding people, things, and social environment. The FFFSI consists of 25 (respondents are required to select one answer to each question) and 27 (respondents are allowed to select up to two answers) question items. With the aim of determining the level of family functioning, the questions were grouped into three categories: "relationships between the family and each family member" such as parent-child and marital relationships and activities within the family, "relationship between the family and subsystems" including interactions and activities with relatives and other people close to the family, "relationship between the family and society" including family activities in school, the workplace, and other places outside the home, as well as the relationship between the family and social environment, and "difficulties and support in daily life". This scale supports the validity and reliability of the FFFS as a measure of the family functioning ${ }^{[10]}$.

Family functioning, defined as "a family's functions that affect its members and society, which are generated when the family members play their roles” by Hohashi et al. (Methods for the assessment of family functioning: FFFS Japanese version I guidelines ${ }^{[8]}$ ), is considered to consist of internal and external functions, which have influences on the family 
members and society, respectively. A decrease in family functioning is defined as "a state of a family in which one or more family members do not play their expected behavioral roles in relation to the family functioning, as recognized by the members", or declined internal and external family functions. "The relationships between the family and each family member", one of the functions, refer to activities within a family performed by its members, including parents, children, and married couples. "The relationship between the family and subsystems" represents relations and activities involving friends, acquaintances, and relatives who interact with a family. "The relationship between the family and society" involves family activities in school, the workplace, and other places outside the home, as well as the relationship with the social environment. These are external family activities. Regarding the satisfaction level of family functioning, a high score indicates a decrease in family functioning. On the other hand, a high score on the scale of the family functioning importance indicates more emphasis on a specific type of family functioning.

\section{Hypothesis}

The author of the present paper made the following prediction on changes in family functioning for patients undergoing surgery and their spouses: During the period between prior to surgery and until patients overcome a crisis situation, patients and their families would strengthen family bonds, which would increase the satisfaction level of family functioning associated with "the relationships between the family and each family member"; the patients and their families would turn their mind to other people in step with improvements in the patients' conditions, and there would be an increase in the satisfaction level of family functioning associated with "the relationship between the family and subsystems" and "the relationship between the family and society", in this order, followed by a complete recovery. Figure 1 shows framework of this study.

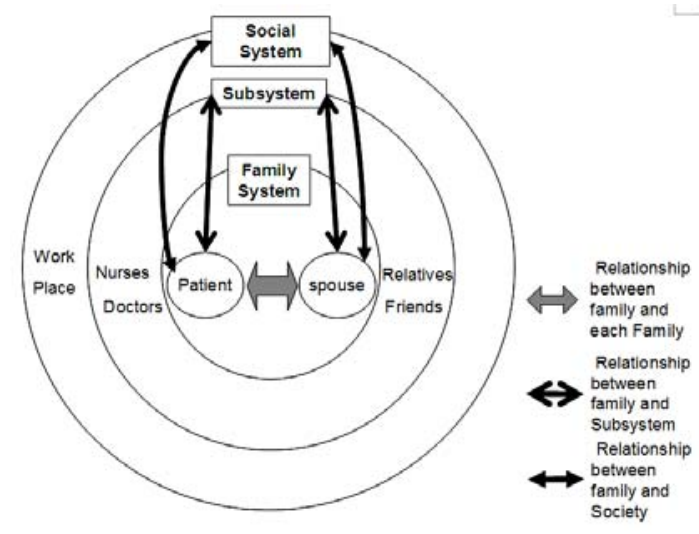

Figure 1. Framework of Family functioning for patients and their spouses

\section{Purpose}

The present study involved patients undergoing surgery under general anesthesia for the first time and their families. Its purpose was to examine changes in family functioning during the period between when a patient has been informed of the necessity of undergoing surgery using general anesthesia and one month after the surgery.

\section{Research methods}

\subsection{Selection of subjects}

Subjects were nine pairs of patients and spouses who satisfied the following conditions: (1) patients undergoing surgical procedures under general anesthesia for the first time and their spouses (a spouse in this study is defined as a person who 
actually plays the role of the spouse as recognized by the patient, regardless of legal or common-law marriage), (2) key persons for patients, (3) patients with the ability to communicate verbally, and (4) persons aged 20 years or older.

\subsection{Data collection}

Patients undergoing surgery under general anesthesia and their families underwent the FFFS (Feetham Family Functioning Survey) Japanese version I (FFFSI) prior to and one month after the surgery. Necessary information, including the background of the subjects and patients' medical condition, was obtained from medical charts on an as-required basis. Data were collected between April 2011 and April 2012.

\subsection{Data analysis}

After tallying up the data, patients and their spouses were divided into two groups, and longitudinal comparative analysis of each group was conducted to examine changes in family functioning. Non-parametric methods, including the Mann-Whitney $U$ and Friedman tests, were used to compare data between the two groups of patients and spouses, and examine changes in family functioning over time.

\section{Ethical considerations}

The subjects participated in the present study based on their own free will. They received written and oral explanations of research objectives and methods, the protection of personal information, and that they could withdraw from the study at anytime, and written consent signed by patients and their spouses or agents was obtained. All data, including information obtained from the questionnaire survey and medical charts, were numbered for identification, stored in a locked cabinet, and strictly managed under the responsibility of the researcher. The study underwent screening by the ethics committees of Meiji University of Integrative Medicine, to which the researcher belongs to, and Research partnership institutions, and was conducted with their approval.

\section{Results}

Although consent was obtained from nine pairs of patients and spouses, patients who developed complications following the surgery and patients and their families who withdrew before the completion of the study were excluded, and four couples in their 50s to 80s were analyzed. All four pairs were patients who underwent cancer surgery for the first time and their parents. Among four patients, three patients were male. Among four patients and spouses, two couple had job and had children who was a minor. Table 1 shows their backgrounds. Figure 2-4 shows main results.

Table 1. Background of Patients and families

\begin{tabular}{|c|c|c|c|c|}
\hline \multicolumn{5}{|c|}{ Patients } \\
\hline No & A & $\mathrm{B}$ & $\mathrm{C}$ & $\mathrm{D}$ \\
\hline Age & $50 \mathrm{~s}$ & $50 \mathrm{~s}$ & $60 \mathrm{~s}$ & $70 \mathrm{~s}$ \\
\hline Gender & Male & Male & Male & Female \\
\hline Type of disease & Rectom Cancer & Stomach Cancer & Breast Cancer & Stomach Cancer \\
\hline Type of surgery & $\begin{array}{c}\text { Laparoendscopic Low } \\
\text { abterior gresection }\end{array}$ & $\begin{array}{l}\text { Laparoendscopic } \\
\text { pylorogastectomy }\end{array}$ & $\begin{array}{c}\text { Mastectomy (sentinel } \\
\text { lymph node) }\end{array}$ & $\begin{array}{l}\text { Laparoendscopic } \\
\text { pylorogastectomy }\end{array}$ \\
\hline Occupation & Yes & Yes & No & No \\
\hline \multicolumn{5}{|c|}{ Spouses } \\
\hline No & A & B & $\mathrm{C}$ & $\mathrm{D}$ \\
\hline Age & $40 \mathrm{~s}$ & $50 \mathrm{~s}$ & $60 \mathrm{~s}$ & $70 \mathrm{~s}$ \\
\hline Gender & Female & Female & Female & Male \\
\hline Occupation & Yes & No & No & No \\
\hline $\begin{array}{l}\text { Have children (a } \\
\text { minor) }\end{array}$ & Yes & Yes & No & No \\
\hline
\end{tabular}


Figure 2. The Score for the importance of Family Functioning and The Score for the sufficiency of family functioning

Figure 3. The Score for the Importance of Family Functioning : Patients

Figure 4. The Score for the Importance of Family Functioning : Families
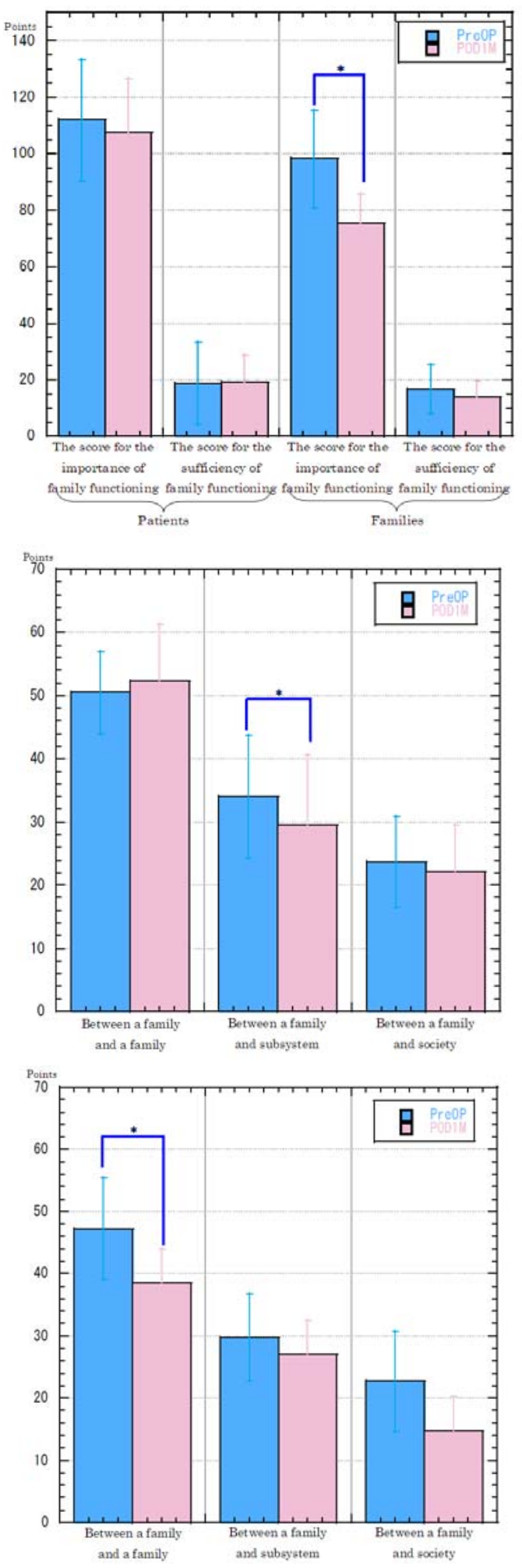


\title{
Changes in family functioning for patients undergoing surgery for the first time and their families
}

\author{
Changes in family functioning for patients
}

One month after surgery, the importance level of family functioning for all patients was lower compared to before surgical treatment, although the differences were not significant. There was no significant difference in the satisfaction level of family functioning between the periods before and one month after surgery. Regarding the three types of satisfaction and importance levels associated with the relationships between the family and: "each family member", "subsystems", and "society", the importance level associated with "the relationship between the family and subsystems" one month after surgery was significantly lower $(\mathrm{P}=0.04)$. The satisfaction level associated with "the relationship between the family and society” one month after surgery was higher compared to before surgical treatment.

\section{Changes in family functioning for patients' spouses}

For patients' spouses, there was no significant difference between the satisfaction level of family functioning determined prior to and one month after surgery, although the importance level of family functioning one month after surgery was significantly lower compared to before the surgical procedures $(P=0.04)$. Regarding the three types of satisfaction and importance levels associated with the relationships between the family and: "each family member", "subsystems", and "society", the importance level associated with "the relationships between the family and each family member" one month after surgery was significantly lower $(P=0.04)$. No significant differences were noted in the other types of family functioning.

\section{Discussion}

\section{1) Changes in family functioning for patients undergoing their first surgery}

There was no significant change in the satisfaction level of family functioning. The author of the present paper made the following prediction on changes in family functioning for patients undergoing surgery and their spouses: During the period between prior to surgery and until patients overcome a crisis situation, patients and their families would strengthen family bonds, which would increase the satisfaction level of family functioning associated with "the relationships between the family and each family member”; the patients and their families would turn their mind to other people in step with improvements in the patients' conditions, and there would be an increase in the satisfaction level of family functioning associated with "the relationship between the family and subsystems" and "the relationship between the family and society", in this order, followed by a complete recovery. In fact, there was no significant changes in family functioning related to patients during the period between prior to and one month after surgery. This was presumably because patients scheduled for hospitalization and surgery attempted to maintain the existing family functioning, and, as a result, the function continued to operate.

For patients undergoing cancer surgery for the first time, the level of importance of family functioning was lower following the surgery. In other words, patients had placed more emphasis on family functioning before surgery was conducted, compared to after the surgical treatment. These patients felt shocked twice by being diagnosed with cancer and the fact they required surgical treatment. They were required to cope with a serious event in their lives that they had never experienced before. As a family is considered to have a mental health function, which helps family members consult with each other regarding worries and personal problems ${ }^{[8]}$, the patients presumably attempted to overcome the crisis situation. However, before surgery was conducted, the level of importance of family functioning associated with "the relationship between the family and subsystems", rather than "the relationships between the family and each family member", was significantly higher, compared to one month after the surgical treatment. Since a subsystem in this study is defined as persons who significantly affect or interact with patients, health care professionals are considered to have served as a subsystem for patients undergoing surgery in hospital. The subjects of the study, or patients diagnosed with cancer and 
scheduled for undergoing surgery for the first time were in a crisis situation, and it was difficult for them and their families alone to overcome it. In this case, as patients had to leave their fate in the hands of medical professionals, they placed an emphasis on "the relationship between the family and subsystems". This means that health professionals are required to establish relationships with patients and provide appropriate intervention, including a proper explanation of treatment and support, for them, in addition to support provided by their families.

\section{2) Changes in the importance of family functioning for the spouses of patients undergoing surgery for the first time}

For the spouses of patients undergoing surgery for the first time, the importance level of family functioning prior to surgery was significantly higher than that after it. If patients are diagnosed with cancer and scheduled to receive surgical treatment for the first time in their lives, their spouses will also be shocked and face a crisis. Patients' spouses had placed more emphasis on family functioning before patients underwent surgery - the period in which the spouses felt shocked. For this reason, the level of importance of family functioning associated with "the relationships between the family and each family member" was significantly higher. In other words, they placed an emphasis on functions that operate according to the relationship between a family and its members. A family is considered to have a mental health function, which helps family members consult with each other. When patients were diagnosed with cancer that required surgery for the first time and their spouses faced a crisis, the spouses attempted to help each other with patients and other family members, or placed importance on "the relationships between the family and each family member". Kawakami et al. ${ }^{[7]}$ analyzed mutual relationships in families living with elderly patients who had been diagnosed with cancer that required surgery, and suggested that the families underwent the following process: (1) they first became anxious over the fact that patients had cancer and required surgery and tried to accept it; (2) they confirmed their roles; and (3) they strengthened their bonds with each other. These results suggest that it is important to provide patients and their spouses with support to help them properly address the crisis, confirm their roles, and support each other.

A study by Starzomski, Rosalie et.al ${ }^{[10]}$ on family functioning adjustment to kidney transplantation did reflect similar areas of importance and similar areas of satisfaction to clear that spouse of pre-emptive patients ware somewhat less satisfied with family functioning than spouse of patients on dialysis pretransplant. It is unclear why this difference existed, however, it may relate to the adjustment spouse were making to the fact that their partner was now seriously ill and being assessed for a transplant, whereas other spouse had already experienced life with their partner on dialysis and had made some adjustments to living with a partner with a chronic illness. It clear that illness disrupts family functioning and relationships regardless of whether it is a spouse is ill. In this study, there was no significant change in the satisfaction level of family functioning for patients' spouses - an index of family functioning. This suggests that, even when spouses faced the crisis of patients undergoing surgery for the first time, the family functions were maintained. This was presumably because patients and their spouses supported each other in a crisis situation to continue their familial relationships and daily lives.

\section{Conclusion}

Prior to surgery, patients undergoing it for the first time and their spouses had placed more importance on family functioning compared to one month after it. In particular, patients and their spouses placed an emphasis on "the relationship between the family and subsystems" and "the relationships between the family and each family member", respectively. Therefore, when cancer patient receive surgical treatment for the first time, it is important for health care professionals to establish appropriate relationships with them, and nurses, in particular, should provide intervention over a long period of time, including a proper explanation of treatment and support. To this end, medical staffs are required to cooperate with each other. It is also important to help patients' spouses accept the crisis situation, in which the patients receive their first cancer treatment, confirm their roles, and support the patients. 
The FFFSI used in the study, designed to determine the importance levels of family functioning and satisfaction in a simple manner, is considered to be a useful tool for providing family members with nursing intervention. However, it is difficult to explain the purpose of the FFFSI to late-middle-aged and young elderly patients undergoing surgery or in a crisis situation, and use it in clinical settings, because the survey involves their privacy.

\section{Limitations of the study}

Since the present study only involved the digestive system and male breast cancer, and the number of subjects was small, it is difficult to generalize the study results, although they may suggest a certain level of tendency. Although the study assumed that the postoperative conditions of patients would become stable one month after surgery, only some patients returned to society, whereas other did not completely recover in terms of appetite and physical strength within the period. Therefore, it is necessary to review the period for examining changes in family functioning, e.g., around the period of discharge from the hospital, and increase the number of subjects.

\section{Reference}

[1] Statistics database of the Ministry of Health, Labour, and Welfare: (static and dynamic) surveys of health care institutions / hospital reports. Available from: http://www.mhlw.go.jp/toukei/saikin/hw/iryosd/08/index.html (6 February 2013, date last accessed).

[2] Takashima N, Itukida K. The Present Status of Perioperative Nursing in the Digestive Organ Surgery under Hospital-days Shortening-Recognition of the Head Nurses by National Investigation. Journal of Japan Academy of Critical Care Nursing. 2009; 5(2): 60-68.

[3] Morioka K, Mochizuki T. Change of a Family Function, New family sociology, ed. Tokyo: baihuukan. 1997: 169-178.

[4] Kinoshita K, Hosaka E, Sonoi Y, A. New version of “Family Sociology” - Basics and Application, ed. Fukuoka. Kyushu University Press. 2008: 13-16.

[5] Hiratani Y, Hohashi N. Family Functions of Child-rearing Single-parent Families in Japan: A Comparison Between Single-parent Families and Pair-matched Two-parent Families: Japanese Journal of Research in Family Nursing. 2010; 16(2): 56-70.

[6] Maekubo K, Okamoto K, Hashimoto M. The effect on family function of home caregivers nursing frail elderly (Part 2): Investigation of marital relationships using Japanese Version I of the Feetham Family Functioning Survey. 2007. Journal of KIBI International University, School of Health Science. 2007; 12: 31-38.

[7] Kawakami Y, Oomachi Y. Intrafamily Interaction Process of Aged Patients' Family after being told Truth as to Diagnosis of Cancer -A Qualitative Analysis 4 Families at Pre and Post Surgical Period-:Journal of Nursing, Shiga University of Medical Science. 2004; 2(1): 23-34.

[8] Starzomski, Rosalie Hilton, Ann; Kleindienst, Martha J. Patient and family adjustment to kidney transplantation with and without an interim period of dialysis / Commentary and response. Nephrology Nursing Journal. 2000; 27(1) : 17-8, 21-32. PMid:10852688

[9] Hohashi N. Assesment of family functioning guide of FFFS Japanese Version: EDITEX, 2008.

[10] Hohashi N., Maeda M., Sugishita C. Development of the Japanese-language Feetham Family Functioning Survey (FFFS) and evaluation of its effectiveness. Japanese Journal of Research in Family Nursing. 2000; 6(1), 2-10. 